INSTITUT NATIONAL DE LA STATISTIQUE ET DES ETUDES ECONOMIQUES

Série des Documents de Travail du CREST

(Centre de Recherche en Economie et Statistique)

\author{
$n^{\circ}$ 2005-37 \\ Le Contrôle des concentrations \\ en France : \\ Une analyse empirique des avis \\ du Conseil de la Concurrence*
}

L. JANIN ${ }_{1}$ - B. MENONI 2

Les documents de travail ne reflètent pas la position de l'INSEE et n'engagent que leurs auteurs.

Working papers do not reflect the position of INSEE but only the views of the authors.

\footnotetext{
* Nous remercions tout particulièrement Karine Brisset, Philippe Choné, Bertin Lalanne, Thierry Magnac, Lucie Muniesa, Anne Perrot, Sylvaine Poret, Bernard Salanié, Saïd Souam ainsi que nos deux rapporteurs pour les conseils qu'ils nous ont prodigués et les remarques qu'ils nous ont adressées.

1 CREST-LEI, 28 rue des Saints-Pères, 75007 Paris. France. Tel. : +33 1445827 55. Fax : +33 144582772. Mail : janin@ensae.fr (Auteur chargé de la correspondance).

2 CREST et EUREQua. (CREST, 15 bld Gabriel Péri, 92245 Malakoff cedex, France). Mail : menoni@ensae.fr
} 


\title{
Le Contrôle des concentrations en France : une analyse empirique des avis du Conseil de la concurrence*
}

\author{
Lionel JANIN ${ }^{\dagger} \quad$ Benoît MENONI ${ }^{\ddagger}$
}

Décembre 2005

*Nous remercions tout particulièrement Karine Brisset, Philippe Choné, Bertin Lalanne, Thierry Magnac, Lucie Muniesa, Anne Perrot, Sylvaine Poret, Bernard Salanié, Saïd Souam ainsi que nos deux rapporteurs pour les conseils qu'ils nous ont prodigués et les remarques qu'ils nous ont adressées.

${ }^{\dagger}$ Auteur chargé de la correspondance, CREST-LEI, 28, rue des Saints-Pères, 75007 Paris, France; tél. : +33144582755, Fax : +33144582772; janin@ensae.fr

${ }^{\ddagger}$ CREST-EUREQua, 15, bd Gabriel Péri, 92245 Malakoff Cedex, France; menoni@ensae.fr 


\begin{abstract}
Merger control is one important tool of competition policy. Economic theory provides some guidance about what criteria to use for the assessment of proposed mergers, mainly the balance between potential efficiency gains (pro competitive effect) and the elimination of a rival (anti competitive effect) in horizontal mergers, but the actual enforcement lies in the hands of competition authorities.

Following previous work by Khemani and Shapiro (1993) and Bergman, Jakobsson, and Razo (2005), we focus on the factors explaining the conclusions of the competition authorities.

We use the complete set of opinions of the French Competition Council (Conseil de la concurrence) in merger cases between 1988 and 2002, to which we apply a latent variable decision model. We use the factors mentioned within the opinions of the Council as explanatory variables, acknowledging a potential endogeneity issue. In a first specification, barriers to entry and buyer power are shown to be the main justifying elements mentioned in the opinions. In a second specification excluding some endogenous factors, market shares and the involved sector appear as more objective determinants of the opinions, but with a low explanatory power. In addition, we also compare a few opinions of the Council with the reaction of the stock market at the announcement of the merger using an event study methodology.
\end{abstract}

Key words: competition policy, merger control.

JEL Classification: D40, K20, L40, L49.

\title{
Résumé
}

Le contrôle des fusions et acquisitions - ou concentrations - constitue l'un des piliers de la politique de la concurrence. La théorie économique fournit des indications sur les critères qui peuvent être utilisés pour évaluer une opération de concentration du point de vue du bien-être social, principalement la tension entre les gains d'efficacité potentiels et la disparition d'un concurrent lors de concentrations horizontales, mais l'application de ces critères reste largement à la discrétion de l'autorité de concurrence.

Dans le prolongement des travaux de Khemani et Shapiro (1993) et de Bergman, Jakobsson, et Razo (2005), nous nous intéressons aux facteurs qui expliquent les conclusions rendues par les autorités de concurrence.

Nous nous appuyons sur l'ensemble des avis en matière de concentration rendus par le Conseil de la concurrence entre 1988 et 2002, pour lequel nous développons un modèle de décision à variable latente. Nous utilisons comme variables explicatives les facteurs mentionnés par le Conseil dans sa décision, tout en soulignant les problèmes d'endogénéité potentiels. Une première spécification met en évidence l'importance des barrières à l'entrée et de la puissance d'achat comme élément de justification par le Conseil de ses avis. Une deuxième spécification montre que les parts de marché ainsi que le secteur sont des déterminants plus objectifs des avis, avec cependant un faible pouvoir explicatif. En outre, nous comparons l'avis du Conseil avec la réaction des marchés financiers à l'annonce de quelques opérations, en esquissant une étude d'événement.

Mots clé : politique de la concurrence, contrôle des concentrations.

Classification JEL : D40, K20, L40, L49. 


\section{Introduction}

Les mouvements de «concentration» des entreprises, qu'ils prennent la forme de fusion, d'acquisition ou de prise de participation, font partie des réorganisations nécessaires au bon fonctionnement d'une économie de marché. Ces opérations, faisant intervenir le plus souvent une entreprise acquéreuse et une entreprise cible, sont motivées par les bénéfices privés attendus, ce qui justifie l'intervention de la puissance publique pour contrôler les opérations qui pourraient avoir un impact négatif sur le «surplus social».

Notre objectif est de préciser les variables intervenant dans les analyses des autorités de concurrence, dans la ligne des travaux qui ont été effectués au Canada par Khemani et Shapiro (1993) et sur les décisions de la Commission européenne par Bergman, Jakobsson, et Razo (2005). Pour ce faire, nous nous appuyons sur l'ensemble des avis du Conseil de la concurrence entre 1988 et 2002, année de l'entrée en application de la loi sur les Nouvelles Régulations Économiques. En France, le contrôle des concentrations dépend du ministre de l'Économie qui peut autoriser ou interdire une opération. S'il estime qu'une opération présente un risque éventuel pour le bon fonctionnement du marché, il doit solliciter le Conseil de la concurrence pour un avis consultatif. Pour justifier ses conclusions, ce dernier retient différents arguments provenant d'une part de l'analyse économique, d'autre part de principes énoncés par la loi. Le but de notre étude consiste à expliquer les avis du Conseil en estimant l'importance de ces différents critères.

En nous limitant à ces avis, nous avons constitué une base de données homogène sur les cas importants de concentration en France. Les variables retenues recouvrent à la fois des grandeurs «objectives » indépendantes de l'avis, comme des chiffres d'affaires, et des critères sur l'appréciation desquels le Conseil dispose d'une certaine liberté et qui risquent donc d'être biaisés, causant par-là même des problèmes d'endogénéité.

En ayant recours à un modèle économétrique à variable latente, nous déterminons l'importance relative des facteurs expliquant les avis du Conseil. Dans un premier temps, nous estimons un modèle descriptif précisant les principaux facteurs mentionnés par le Conseil pour justifier ses avis. Dans un second temps, nous estimons un modèle explicatif des conclusions en nous restreignant à des variables supposées exogènes.

Notre étude met en évidence l'importance de la puissance d'achat et des barrières à l'entrée comme variables explicatives des avis du Conseil de la concurrence alors que l'identité des personnes en charge du cas n'est pas significative. Si on exclut les variables endogènes, les parts de marché retrouvent un pouvoir explicatif des conclusions, quoique limité.

L'article adopte le plan suivant : après avoir rappelé un certain nombre de résultats de la théorie économique sur le sujet, nous présentons la politique de la concurrence en France en matière de concentration. Nous décrivons ensuite les données que nous utilisons puis nous développons le modèle économétrique et les différentes spécifications retenues. Compte tenu du faible nombre d'observations dont nous disposons, nous présentons également des tests sur la robustesse de nos estimations, par des procédures de ré-échantillonnage. Nous terminons enfin par une analyse de quelques cas de concentration pour lesquels nous pouvons identifier une réaction spécifique des marchés financiers à l'annonce de l'opération, avant de conclure. 


\section{1 Éléments de théorie économique}

\subsection{Concurrence et efficacité}

Pourquoi assurer la promotion de la concurrence? Selon la théorie économique, la concurrence n'est pas recherchée comme une fin en soi, mais comme un moyen de parvenir à l'efficacité économique. D'une part elle permet la diminution des prix, jusqu'au coût marginal de production en situation de concurrence parfaite, avec érosion de la rente des entreprises (efficacité allocative); d'autre part, elle incite les entreprises à rechercher des réductions de coût ou des améliorations de la qualité pour conquérir des parts de marché ou augmenter leur marge, ce qui conduit à l'utilisation efficace des techniques de production disponibles et à la recherche de l'innovation (efficacité productive).

Une entreprise peut cependant se retrouver en situation de monopole parce qu'elle produit plus efficacement que ses concurrentes potentielles, en particulier si les entreprises se font concurrence par l'innovation. De tels marchés peuvent cependant être dynamiquement concurrentiels, et correspondre à une dynamique schumpétérienne. Il existe également des monopoles naturels lorsque la fonction de coût est sous-additive ou lorsque l'on souhaite éviter la duplication d'une infrastructure coûteuse, typiquement dans les industries de réseau. De fait, le droit de la concurrence ne prévoit d'intervention de la puissance publique qu'en cas d'abus de position dominante.

\subsection{Le marché pertinent}

Une opération de concentration risque de poser des problèmes de concurrence principalement dans le cas où les entreprises concernées produisent des biens substituables. On parle dans ce cas de concentration horizontale. En pratique, on a recours à la notion de marché pertinent qui regroupe « l'ensemble des biens et services au sein duquel il est utile de veiller à ce que règne une concurrence suffisante, parce que la proportion de consommateurs susceptibles de se reporter vers d'autres biens ou services, en cas de hausse des prix, est trop faible pour dissuader les offreurs de pratiquer de telles hausses ${ }^{1} \gg$. Le marché pertinent, qui est défini par rapport à la demande, est le résultat de la recherche de l'ensemble des produits qui exercent une contrainte concurrentielle sur les produits concernés par l'opération, ce qui peut se faire selon la méthode théorique du monopoleur hypothétique ou test SSNIP (Small, but Significant, Non-transitory Increase in Price)

Alors que la détermination du marché pertinent est une nécessité pratique, elle n'est pas indispensable à une analyse économique, qui s'appuie sur la demande des consommateurs caractérisée par exemple par les élasticités de substitution entre produits.

\subsection{Le pouvoir de marché}

Une concentration a en général deux effets. D'une part, elle peut conduire à des gains d'efficacité, sous la forme d'une diminution du coût de production, d'une amélioration de la qualité, de l'introduction de nouveaux produits, etc.

\footnotetext{
1 Acquisition de certains des actifs du groupe Benckiser par le groupe Sara Lee, avis $\mathrm{n}^{\mathrm{O}}$ 00-A-07.
} 
D'autre part, elle peut permettre aux entreprises d'augmenter leur prix de façon stratégique, ce qui constitue un renforcement du pouvoir de marché. Williamson (1968) est le premier à avoir relevé l'arbitrage qui existe entre ces deux effets, même si son analyse optimiste des gains d'efficacité a été tempérée depuis (voir Mc Fetridge (1996)).

Le renforcement du pouvoir de marché passe par deux mécanismes différents : soit des effets unilatéraux, soit des effets coordonnés. Les entreprises qui prennent part à la concentration cherchent à maximiser leur profit joint $a$ posteriori, typiquement par une réduction de la production et une augmentation de prix. Les autres entreprises du secteur adaptent en conséquence leur comportement : il en résulte un nouveau prix d'équilibre qui traduit les effets unilatéraux de la concentration. Par contre, si les conditions ex post du marché font qu'un comportement coopératif et non plus concurrentiel des entreprises peut se mettre en place, on parle d'effets coordonnés ou de collusion, qui peut être éventuellement tacite.

Effets unilatéraux Farrell et Shapiro (1990) ont fait l'étude générale d'une fusion horizontale dans le cadre de la concurrence en quantité pour des biens homogènes. Dans ce cadre, une fusion sans gain d'efficacité se traduit nécessairement par une hausse de prix et une concentration a un effet positif sur l'ensemble «concurrents et consommateurs » si la part de marché cumulée des entreprises qui fusionnent est inférieure à une somme pondérée des parts de marché des entreprises concurrentes.

Deneckere et Davidson (1985) ont traité le cas de la concurrence en prix. Ils ont montré dans ce modèle qu'en l'absence de synergie une concentration conduit toujours à une augmentation de prix, qu'elle est toujours profitable pour les entreprises qui y prennent part et qu'elle l'est davantage pour celles qui restent extérieures à l'opération.

Ce dernier résultat est assez général si les gains d'efficacité sont faibles. C'est ce qu'on appelle parfois le dilemme de l'insider, qui souhaite qu'une concentration ait lieu mais sans en faire partie.

L'analyse économique cherche à prévoir quelle sera la structure du marché après concentration. Dans les modèles précédents, les acteurs 《extérieurs » avant et après l'opération étaient les mêmes. Cependant, il peut exister des entreprises qui ne sont pas présentes sur le marché mais qui sont susceptibles d'entrer en cas d'augmentation des prix après la concentration. De tels entrants potentiels, par exemple des importateurs étrangers, peuvent constituer une menace concurrentielle capable de restreindre le pouvoir de marché de l'entité issue de la concentration et donc de maintenir une concurrence effective. Cette vue est conforme à la notion de marché contestable développée par Baumol, Panzar, et Willig (1982).

Il peut également exister des barrières à l'entrée qui empêchent l'arrivée de nouveaux concurrents. Les barrières à l'entrée naturelles sont liées aux caractéristiques spécifiques d'un marché, comme la nécessité d'un fort investissement initial, la maîtrise de technologies complexes, l'existence de brevets ou de normes ou l'importance de la réputation. Il peut exister également des barrières à l'entrée stratégiques dues au comportement des entreprises en place. Fudenberg et Tirole (1984) donnent des exemples d'investissements stratégiques d'un monopole pour tenter d'empêcher l'entrée d'un concurrent. 
Effets coordonnés Les situations mentionnées précédemment supposent une indépendance de comportement entre les entreprises. Or l'un des risques associés à un marché très concentré est l'apparition d'une entente au sein de laquelle les entreprises se coordonnent pour pratiquer des prix plus élevés que les prix concurrentiels. De telles pratiques collusives, qu'elles soient explicites ou tacites, sont interdites par le droit de la concurrence, mais sont par nature difficiles à détecter. Aussi le contrôle des concentrations intègre-t-il une dimension préventive, en cherchant à éviter l'apparition de structures de marché favorables à la collusion, qui portent le nom de position dominante collective dans le contexte européen. L'analyse économique a identifié un certain nombre de facteurs favorables à la collusion sur le marché post concentration, entre autres la transparence des prix, l'homogénéité du marché, la symétrie des structures de coût des entreprises ou la présence de capacités de production excédentaires (voir Motta (2004) pour une revue générale et Compte, Jenny, et Rey (2002) pour un exemple précis).

\subsection{Relations verticales}

Les entreprises peuvent ne pas être en contact direct avec les consommateurs mais appartenir à une filière verticale. Selon le mode de concurrence aux différents niveaux de la filière et le degré d'intégration verticale, une fusion peut avoir des effets différents, par exemple sur le risque d'exclusion de concurrents dans le cadre d'une entreprise intégrée verticalement (voir Rey et Tirole (2005)).

Le pouvoir de négociation des entreprises lors du partage du surplus dans une relation verticale (la «puissance d'achat ») dépend de la structure concurrentielle et peut être modifié lors d'une concentration. En particulier différents modèles mettent en évidence que le risque de fusion engendrant des pertes de surplus social est plus important en aval qu'en amont (voir Allain et Souam (2004)) .

Les éléments de la théorie économique mis en avant jusqu'ici sont partiellement intégrés dans la législation relative à la politique de la concurrence. Si on observe des rapprochements dans ce domaine entre droit et économie (voir Jenny (1994)), il n'en demeure pas moins que le droit utilise une formulation spécifique, que nous présentons maintenant.

\section{2 Éléments du droit de la concurrence relatifs au contrôle des concentrations}

$\mathrm{Au}$ niveau national, la politique de la concurrence est régie par le droit français, auquel s'ajoute le droit européen, qui lui est supérieur en vertu du principe de la primauté de la règle communautaire. Néanmoins, le principe de subsidiarité prévoit de renvoyer aux États les questions qu'ils sont mieux à même de traiter. 


\subsection{Le droit européen}

L'Union européenne s'est dotée d'un règlement spécifique pour le contrôle des concentrations, le règlement du Conseil no 4064/89 du 21 décembre $1989^{2}$. Ce règlement pose la compétence exclusive de la Commission européenne en matière de concentration pour toute opération présentant « une dimension communautaire » définie par des seuils de chiffre d'affaires ${ }^{3}$. En deçà de ces seuils ou pour des opérations présentant un caractère national, il est prévu de renvoyer le cas soit devant les autorités nationales, soit à la Commission, selon l'autorité qui sera jugée la plus compétente.

\subsection{Le droit français}

Les législations concernant le contrôle de la concurrence sont apparues en Europe peu après la Deuxième Guerre mondiale. En France, elle date de 1953, avant d'être révisée en 1977. La réforme importante qui nous concerne est l'entrée en vigueur de l'Ordonnance $\mathrm{n}^{\mathrm{o}} 86-1243 \mathrm{du} 1^{\mathrm{er}}$ décembre 1986, relative à la liberté des prix et de la concurrence, qui instaure un contrôle spécifique des concentrations et crée le Conseil de la concurrence. Intégrée dans le Livre IV du code du commerce le 18 septembre 2000, cette ordonnance est restée en vigueur jusqu'à la loi sur les Nouvelles Régulations Économiques (NRE), adoptée le 15 mai 2001 et mise en application le 3 mai 2002.

L'article 39 de l'Ordonnance définit une concentration de la manière suivante : «La concentration résulte de tout acte, quelle qu'en soit la forme, qui emporte transfert de propriété ou de jouissance sur tout ou partie des biens, droits et obligations d'une entreprise ou qui a pour objet, ou pour effet, de permettre à une entreprise ou à un groupe d'entreprises d'exercer, directement ou indirectement, sur une ou plusieurs autres entreprises une influence déterminante. » Cette définition recoupe deux critères : le transfert de propriété, droits ou obligations d'une entreprise, et l'acquisition d'une influence déterminante. Cette définition inclut donc la création d'entreprise commune ou les changements importants dans la détention du capital d'une entreprise.

L'article 38 précise les conditions de contrôlabilité de l'opération. Il suffit que l'un ou l'autre des seuils suivants soit atteint pour que l'opération soit contrôlable :

- une condition de chiffre d'affaires : les entreprises parties à l'opération doivent réaliser en France un chiffre d'affaires supérieur à sept milliards de francs, à condition que deux d'entre elles réalisent plus de deux milliards de francs;

- une condition de part de marché : les entreprises parties à l'opération doivent détenir plus de $25 \%$ d'un marché national.

2 Entré en vigueur le 21 septembre 1990, il est maintenant remplacé par le règlement $\mathrm{n}^{\mathrm{o}} 139 / 2004$ du 20 janvier 2004, en application depuis le $1^{\mathrm{er}}$ mai 2004.

${ }^{3}$ Pour avoir une dimension communautaire, une opération de concentration doit dépasser l'un des seuils de chiffre d'affaires (CA) suivants :

- l'entité fusionnée a un CA mondial supérieur à 5 milliards d'euros, et deux entreprises réalisent des CA supérieurs à 250 millions d'euros en Europe, dont pas plus de $2 / 3$ dans un seul pays;

- l'entité fusionnée a un CA mondial supérieur à 2,5 milliards d'euros et un CA supérieur à 100 millions d'euros dans trois pays de l'Union européenne, et deux entreprises ont des CA supérieurs à 100 millions d'euros dans le monde et 25 millions d'euros dans trois pays de l'Union européenne. 
En pratique, la détermination du seuil en part de marché suppose d'avoir déjà défini les marchés pertinents concernés par l'opération.

Les autorités en charge du contrôle des concentrations en France sont d'une part le ministre en charge de l'économie, par l'intermédiaire de la $\mathrm{DGCCRF}^{4}$; d'autre part le Conseil de la concurrence ${ }^{5}$, créé par l'Ordonnance de 1986 et prenant la suite de la Commission de la concurrence.

Si une opération de concentration est contrôlable, elle peut faire l'objet d'une notification au ministre de l'Économie ${ }^{6}$, qui dispose alors d'un délai de deux mois pour saisir pour avis le Conseil de la concurrence. En l'absence de notification, le ministre peut ordonner une enquête jusqu'à trois mois après l'opération. À l'issue de cette première phase (équivalent des phases I américaine ou européenne), le ministre autorise les opérations qui ne sont pas de nature à porter atteinte au fonctionnement concurrentiel du marché. En revanche, s'il émet des réserves, il doit saisir le Conseil (équivalent de la phase II) et dispose d'un délai supplémentaire de quatre mois pour rendre sa décision. En l'absence de réponse des services du ministre de l'Économie, on considère qu'il y a approbation tacite.

Quand le ministre de l'Économie sollicite l'avis du Conseil de la concurrence, ce dernier établit un bilan concurrentiel en vertu de l'article 41. Si ce dernier est positif, l'analyse est terminée. En revanche, si l'opération comporte des risques d'atteinte à la concurrence, le Conseil établit également un bilan économique. L'article 41 précise : «Le Conseil de la concurrence apprécie si le projet de concentration ou la concentration apporte au progrès économique une contribution suffisante pour compenser les atteintes à la concurrence. Le Conseil tient compte de la compétitivité des entreprises en cause au regard de la concurrence internationale. » Le Conseil peut explicitement invoquer l'efficiency defence, c'est-à-dire que des gains d'efficacité peuvent contrebalancer l'effet anticoncurrentiel.

Après avoir reçu l'avis du Conseil, il appartient alors au ministre de rendre la décision finale d'autorisation, avec ou sans conditions, ou d'interdiction.

\section{Présentation des données}

Nous avons étudié les 66 avis complets ${ }^{7}$ que le Conseil de la concurrence a rendus entre avril 1988 et décembre 2002. À l'exception d'un cas $^{8}$, notre base de

\footnotetext{
${ }^{4}$ Direction générale de la concurrence, de la consommation et de la répression des fraudes.

5 Le Conseil se compose de huit membres issus du monde juridique, que ce soit le Conseil d'État, la Cour des comptes ou la Cour de cassation; de quatre membres choisis en raison de leurs compétences économiques, enfin de cinq membres issus de divers secteurs du monde du travail, ayant exercé une activité marchande.

${ }^{6}$ La notification est devenue obligatoire à partir de certains seuils de chiffre d'affaires (150 millions d'euros hors taxes au niveau mondial et 15 millions d'euros pour au moins deux entreprises en France) depuis l'entrée en vigueur de la loi NRE (Art. L. 430-2 du code du commerce). Le seuil de 15 millions d'euros a été relevé à 50 millions d'euros par l'ordonnance 2004-274 du 25 mars 2004.

7 Sur les 73 saisines du Conseil, 7 cas n'ont pas donné lieu à une analyse complète : 2 procédures ont été annulées à la demande de l'une parties - avis $\mathrm{n}^{\circ}$ 88-A-05 et prise de participation de la société Havas dans la société $R M C$ Radio, 1992, qui n'a pas donné lieu à la rédaction d'un avis - et pour 5 cas — avis $\mathrm{n}^{\circ} 90-\mathrm{A}-11, \mathrm{n}^{\circ} 93-\mathrm{A}-16, \mathrm{n}^{\circ} 94-\mathrm{A}-13, \mathrm{n}^{\circ} 94-\mathrm{A}-30$ et $n^{\circ}$ 97-A-14 - le Conseil a constaté que l'opération que lui demandait d'analyser le ministre de l'Économie n'entrait pas dans son domaine de compétence ou d'intervention.

8 Reprise de la société Ouest Répartition Pharmaceutique par la société Alliance Santé Distribution, avis $\mathrm{n}^{\circ} 02-\mathrm{A}-15$.
} 
données couvre ainsi l'activité du Conseil en matière de concentration pendant l'ensemble de la durée d'application de l'Ordonnance de 1986. Quoique la loi NRE ait sensiblement modifié les conditions de saisine du Conseil, nous avons pris le parti d'inclure dans nos données une opération post NRE car il nous a semblé que celle-ci aurait été examinée même si cette disposition législative n'était pas entrée en vigueur.

\subsection{Analyse historique}

La répartition dans le temps des avis rendus par le Conseil est inégale et assez décorrélée de l'activité générale de fusions et acquisitions. La figure 1 montre l'évolution comparée du nombre d'avis rendus, du nombre et de la valeur totale des fusions et acquisitions en France pour la période 1990-2001 ${ }^{9}$. Nous pouvons

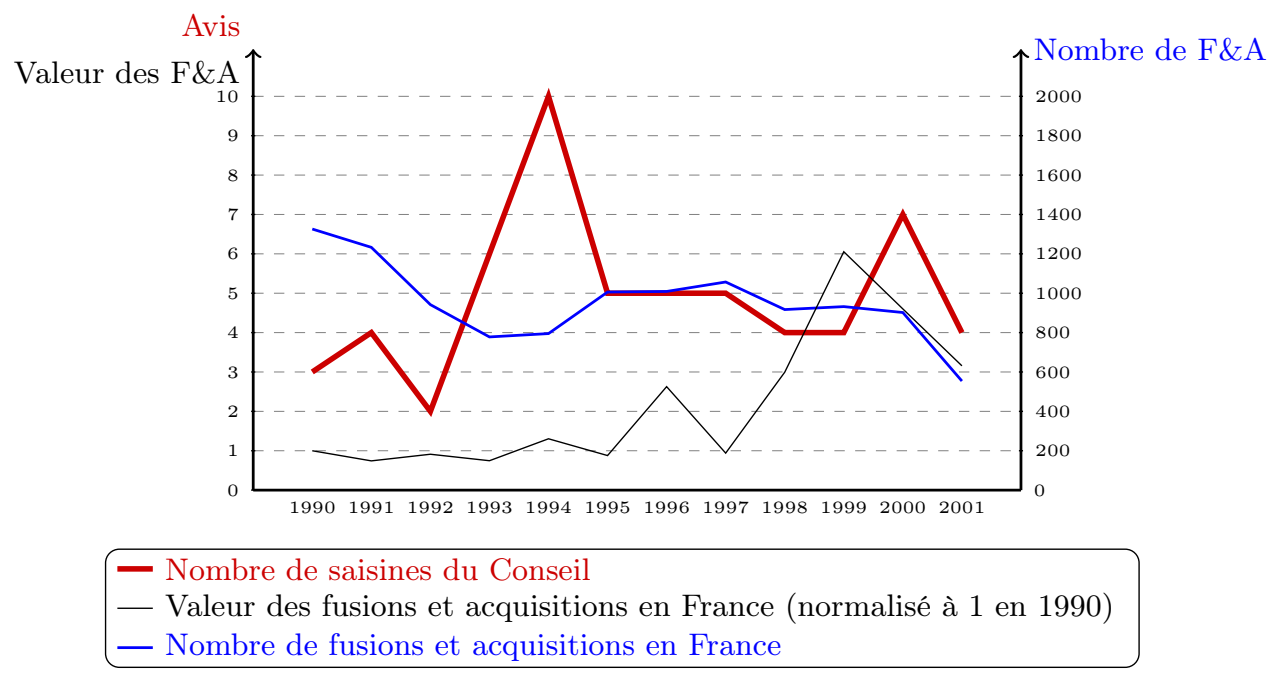

Fig. 1 - Saisines du Conseil, nombre et valeur des fusions et acquisitions entre 1990 et 2001.

distinguer diverses périodes plus ou moins fécondes en opérations de concentration. Outre les stratégies industrielles des firmes, des facteurs macroéconomiques exogènes tels que le Traité de Rome ou une bulle boursière peuvent influencer le comportement des entreprises d'un secteur et par là même le nombre de projets de concentration soumis au ministre au cours d'une année. Le lecteur intéressé trouvera dans Derhy (1995) une analyse claire et détaillée de ces différentes phases de fusions et acquisitions en France.

\subsection{Avis du Conseil de la concurrence et décision du mi- nistre}

Les cas transmis au Conseil de la concurrence ont fait l'objet d'un examen préliminaire par la DGCCRF, qui a suspecté une atteinte à la concurrence. Cependant, dans 30 cas, le Conseil a conclu à l'absence d'atteinte à la concurrence

\footnotetext{
9 Données provenant de Fusions et Acquisitions magazine.
} 
et a suggéré une autorisation. Sur les 36 cas restants, le Conseil a estimé dans 10 cas qu'il existait une contribution au progrès économique susceptible de compenser les risques d'atteinte à la concurrence, ce qui a conduit à une suggestion d'autorisation, assortie éventuellement de conditions. Dans les autres cas, des engagements ont été et si aucun remède n'était pas possible, une interdiction a été proposée.

Au terme de ses analyses, le Conseil a émis 40 avis favorables, 17 avis suggérant une autorisation soumise au respect de certains engagements et 9 avis défavorables. Compte tenu du caractère bicéphale de l'organe de contrôle des concentrations en France, nous pouvons nous demander si les recommandations du Conseil sont globalement suivies ou pas. Le tableau 1 nous permet de répondre à cette question.

\begin{tabular}{lccrr}
\hline \hline & \multicolumn{3}{c}{ Avis du Conseil } \\
\cline { 2 - 4 } Décision finale du ministre & Autorisation & $\begin{array}{c}\text { Autorisation } \\
\text { conditionnelle }\end{array}$ & Opposition & Total \\
\hline Autorisation & 32 & 0 & 1 & 33 \\
Autorisation tacite & 5 & 0 & 0 & 5 \\
Autorisation conditionnelle & 1 & 17 & 4 & 22 \\
Opposition & 2 & 0 & 3 & 5 \\
Opération abandonnée & 0 & 0 & 1 & 1 \\
\hline Total & 40 & 17 & 9 & 66 \\
\hline
\end{tabular}

TAB. 1 - Avis du Conseil et décision du ministre

Nous constatons que le ministre suit globalement les analyses et avis du Conseil, même si certaines divergences peuvent apparaître. Notons que nous n'avons pas eu accès à la lettre du ministre pour cinq avis, tous étant favorables. En outre, une procédure a été annulée après que le Conseil a émis un avis défavorable. Le ministre n'a ainsi pas eu à se prononcer sur ce cas ${ }^{10}$.

\subsection{Construction des variables explicatives}

Nous avons relevé un certain nombre de variables explicatives dans le texte des avis du Conseil.

Ces variables peuvent être divisées en deux classes : d'une part des variables que nous traitons comme « objectives »; d'autre part des variables dont l'appréciation est laissée en partie à la discrétion du Conseil. Ainsi lorsque le Conseil mentionne la présence de barrières à l'entrée, il choisit de le faire pour justifier sa décision. Il est d'ailleurs à noter que les divergences entre le Conseil et la DGCCRF proviennent justement de l'appréciation de ces variables ${ }^{11}$. Étant donné que nous ne disposons pas d'indicateurs objectifs et extérieurs pour ces variables, nous ne sommes pas en mesure de contrôler l'utilisation qu'en fait le Conseil. Il s'agit de variables descriptives mais elles induisent probablement des biais d'endogénéité que nous ne pouvons corriger en l'absence d'instrument.

\footnotetext{
10 La société Chep France a renoncé à acquérir la société Logistic Packaging Return suite à l'avis défavorable du Conseil (avis n ${ }^{0} 00-\mathrm{A}-17$ ).

${ }^{11}$ Dans le cas concernant les entreprises Sara Lee et Benckiser (avis no 00-A-07) le Conseil a rendu un avis favorable en relevant l'absence de barrières à l'entrée, alors que le ministre a interdit l'opération en arguant de la présence de fortes barrières à l'entrée.
} 


\subsubsection{Les variables « objectives » dans l'avis}

Les conditions de chiffre d'affaires Pour chaque dossier, le Conseil examine si les conditions de chiffre d'affaires (seuils absolus) qui déterminent sa compétence sont remplies ce qui arrive dans $23 \%$ des cas. Sur ces 15 avis, on ne relève aucune opposition : l'opération a été autorisée à 7 reprises et le Conseil a demandé que soient pris un certain nombre d'engagements pour 8 cas.

Entreprise défaillante Il se peut que l'une des entreprises concernées par l'opération soit dans une situation financière telle que le maintien de son activité n'est plus possible. Cette situation, dite de l'entreprise défaillante en droit américain, est évoquée dans cinq cas. La modification inévitable de la structure du marché peut modifier l'attitude des autorités de concurrence ${ }^{12}$.

Les marchés pertinents Le nombre de marchés pertinents étudiés est variable et dépend fortement du secteur étudié. Dans $80 \%$ des cas, le Conseil retient entre un et trois marchés pertinents. Les cas extrêmes correspondent à des situations pour lesquelles une définition du marché à un niveau national n'était pas adaptée et pour lesquelles le Conseil a dû recourir à des segmentations géographiques en définissant des marchés «locaux ${ }^{13}$. Dans ce cas, nous n'avons pas retenu pour la part de marché celle qui est calculée au niveau local (souvent des monopoles locaux) mais plutôt celle qui est calculée au niveau national, en marquant cette situation par une indicatrice.

La définition des marchés pertinents est une opération délicate. La méthodologie proposée par les économistes au travers du test SSNIP n'est pratiquement jamais applicable et jamais appliquée pour les cas que nous considérons. En revanche, le Conseil de la concurrence dépend de la jurisprudence, nationale et communautaire, pour la définition des marchés. Nous avons fait l'hypothèse discutable - que les marchés retenus par le Conseil étaient exogènes au processus de décision, en nous appuyant sur l'existence d'une méthodologie établie ${ }^{14}$

Les parts de marché Sur chacun des marchés pertinents qu'il retient, le Conseil indique en général les parts de marché des entreprises parties à la concentration. Nous avons créé trois variables correspondant à la part de l'acquéreur, de la cible et à la part de marché cumulée ${ }^{15}$. Les opérations qui sont traitées par le Conseil ont fait l'objet d'un filtrage par la DGCCRF, qui a estimé qu'elles posaient des problèmes de concurrence, il s'agit donc principalement d'opérations horizontales ${ }^{16}$. Dans quelques cas, les parts de marché ne sont pas mentionnées explicitement, ce qui nous a amené à les reconstruire d'après les données disponibles. Par ailleurs, il arrive fréquemment que les parts de marché soient couvertes par le secret des affaires et que seule une fourchette de $10 \%$ soit disponible. Nous avons retenu dans ce cas le milieu de la fourchette.

\footnotetext{
12 Voir, par exemple, l'acquisition de la Société de Mécanique Magnétique par la société Seiko-Seiki Belgium, avis no 96-A-01.

13 Voir l'acquisition de la société Cedest par la société Holdercim, avis n ${ }^{\circ}$ 94-A-24 ou l'acquisition du groupe GTM par la société Vinci, avis $\mathrm{n}^{\circ}$ 01-A-08.

14 Voir l'étude du Conseil à ce sujet dans le rapport annuel de 2001 http://www. conseil-concurrence.fr/html/article_58.htm.

${ }^{15}$ Les variables part de marché sont continues et comprises entre 0 et 1.

16 Dans six cas, l'acquéreur n'est initialement pas présent sur le marché.
} 
Malheureusement, le Conseil ne donne pas de manière systématique les parts de marché des entreprises concurrentes, ce qui interdit de calculer des indices de concentration comme l'indice de Herfindahl-Hirschman.

Nous avons considéré que les parts de marché étaient des variables exogènes à la décision, mesurées le plus souvent par le chiffre d'affaires des entreprises sur le marché considéré.

Nous avons également rajouté une variable indicatrice monopole si la part de marché cumulée des parties est supérieure à $90 \%$. Cette variable repère les situations où la concentration aboutit à une quasi-monopolisation du secteur. Elle a pour but de détecter des effets non linéaires des parts de marché.

Choix du marché pertinent de référence Nous avons choisi de ne retenir, pour chacun des cas étudiés, que le marché le plus défavorable pour les entreprises. Nous avons en effet postulé que le Conseil se référait implicitement à ce marché car c'est a priori sur celui-ci que les pratiques anti-concurrentielles vont le plus facilement voir le jour. Nous avons retenu celui pour lequel la somme des parts de marché des parties est la plus élevée en nous restreignant, le cas échéant, aux marchés sur lesquels le Conseil a identifié des risques d'atteinte à la concurrence, aux marchés sur lesquels ces risques ne sont pas compensés par une contribution au progrès économique ou pour lesquels il existe des barrières à l'entrée.

\subsubsection{Les variables de contexte}

Nous avons rajouté plusieurs variables correspondant soit au secteur soit aux personnes en charge du dossier lors de la décision.

Les variables de secteur Nous avons inclus une indicatrice sectorielle, distinguant six secteurs ${ }^{17}$ : secteur agricole et industrie agro-alimentaire, industrie manufacturière, infrastructures (regroupant les secteurs du bâtiment, des travaux publics, de la distribution d'eau et d'énergie), les services, desquels sont séparés le secteur de la distribution et celui des médias (voir l'annexe A.4).

Avec l'idée de disposer d'un instrument pour le pouvoir de négociation, nous avons également inclus une variable grande distribution indiquant si un produit concerné par la concentration est vendu par l'intermédiaire de la grande distribution.

Les variables de personnalité Nous avons inclus un certain nombre de variables décrivant les personnes impliquées dans le processus de décision : identité du président de séance, identité du ministre, une variable indicatrice droite précisant si le ministre est de droite au moment de l'opération ${ }^{18}$.

Il est à noter que l'effet de l'identité du ministre peut jouer à deux niveaux. D'une part le ministre intervient dans la première phase du contrôle des concentrations, dans la mesure où la DGCCRF choisit les cas qu'elle défère au Conseil de la concurrence. Ensuite il prend la décision finale, à partir de l'avis du Conseil. Cependant, l'identité du Ministre ne devrait en principe pas jouer sur l'avis du Conseil, qui est indépendant.

\footnotetext{
17 Nous avons également testé une décomposition plus fine des secteurs (NAF 17) mais elle ne donne pas de résultats significatifs.

18 Ces indicatrices politiques se confondent avec des indicatrices temporelles.
} 
Concurrent équivalent Le Conseil s'intéresse également à la structure de la concurrence à la suite de l'opération. En l'absence d'indicateur systématique des parts de marché des concurrents, le Conseil relève parfois la présence d'un concurrent équivalent, c'est-à-dire une entreprise dont la structure (groupe multinational par exemple), les parts de marché, la notoriété sont équivalentes à celle de l'entité issue de la fusion. Nous avons relevé 20 cas de ce type.

\subsubsection{Les variables potentiellement endogènes dans l'avis}

Le bilan concurrentiel Dans chacun de ses avis, le Conseil établit un bilan concurrentiel, précisant s'il existe des risques d'atteinte à la concurrence, et dans ce dernier cas si ces risques peuvent être compensés par une contribution au progrès économique, conformément à l'article 41 de l'Ordonnance de 1986. Le bilan concurrentiel est consubstantiel à la décision du Conseil et donc totalement endogène.

La puissance d'achat Lorsqu'un secteur se trouve intégré dans une chaîne verticale comprenant des fournisseurs ou éventuellement des entreprises clientes, les relations entre les deux niveaux se fait le plus souvent par l'intermédiaire d'une négociation. Si l'un des secteurs est suffisamment concentré pour disposer d'un pouvoir de négociation important, on parle de puissance d'achat (ou de puissance de vente dans le cas d'une dépendance par rapport à ces fournisseurs). L'exemple le plus important est celui de produits vendus par l'intermédiaire de la grande distribution, aujourd'hui fortement concentrée. Dans ce cas, le Conseil considère souvent que les entreprises fournisseurs dépendent de la grande distribution qui est en mesure d'empêcher toute augmentation de prix suite à une concentration. Comme le montre l'analyse divergente de l'appréciation de la puissance d'achat dans le cas $\mathrm{n}^{\circ}$ 04-A-16 concernant le rachat de Moulinex par $S e b$ entre le Conseil et le ministre, la puissance d'achat ne fait pas l'objet d'une mesure objective mais résulte essentiellement d'une appréciation de la situation par le Conseil, et présente sans doute des problèmes d'endogénéité.

Les barrières à l'entrée Nous avons créé une indicatrice correspondant aux situations dans lesquelles le Conseil fait explicitement mention de telles barrières, ce qui survient dans vingt-six cas. Nous avons pris le parti de ne pas distinguer les barrières d'ordre technologique des barrières d'ordre financier, institutionnel, etc. L'exemple précédemment mentionné de la divergence d'avis entre le Conseil et le ministre sur la présence de barrière à l'entrée dans le cas Sara Lee-Benckiser illustre la difficulté à mesurer objectivement leur présence.

Collusion Le risque de collusion dans un marché concentré est l'un des problèmes soulevé par la théorie économique. Le Conseil mentionne la présence ou le risque de collusion sur le marché considéré dans six avis. 


\section{La modélisation économétrique}

\subsection{Régression logistique}

La variable de conclusion est une variable discrète prenant trois modalités : avis favorable, avis favorable sous conditions et avis défavorable. En conséquence, nous estimons des modèles de choix discret du type logit polytomique ordonné. Nous avons également testé un modèle de choix non ordonnés dont les résultats sont présentés dans l'annexe B.3.

Formellement, nous supposons qu'il existe une variable latente $Y^{*}$, à laquelle nous n'avons par définition pas accès mais qui peut être expliquée par les variables dont nous disposons. En outre, il existe deux seuils $s_{1}$ et $s_{2}$ tels que :

- si $Y^{*}<s_{1}$, alors le Conseil autorise l'opération,

- si $s_{1} \leq Y^{*}<s_{2}$, alors le Conseil autorise l'opération mais la soumet à des conditions,

- si $Y^{*} \geq s_{2}$, alors le Conseil interdit l'opération.

Si $X$ désigne la matrice des réalisations des variables explicatives dont nous disposons, nous postulons que l'indice $Y^{*}$ peut être linéairement expliqué par $X$, autrement dit la relation $Y^{*}=X \beta+u, \beta$ désignant le vecteur des poids attribués à chacune des explicatives et $u$ un terme d'erreur suivant une loi logistique dans le modèle logit.

Si nous notons $F$ la fonction de répartition de la variable $u$, alors :

- le Conseil autorise l'opération avec la probabilité $F\left(s_{1}-X \beta\right)$,

- le Conseil autorise l'opération en la soumettant à des conditions avec la probabilité $F\left(s_{2}-X \beta\right)-F\left(s_{1}-X \beta\right)$,

- le Conseil interdit l'opération avec la probabilité $1-F\left(s_{2}-X \beta\right)$.

Autrement dit, si $X \beta$ est élevé, le Conseil aura tendance à interdire l'opération ; au contraire si $X \beta$ est petit, il aura tendance à autoriser l'opération.

Cette modélisation peut recevoir deux interprétations différentes. Si les régresseurs sont exogènes, elle peut être vue comme un modèle de choix discret par maximisation de l'utilité, sous l'hypothèse que les résidus suivent une loi des valeurs extrêmes. Il s'agit dans ce cas d'un modèle ayant un pouvoir prédictif. Dans l'hypothèse où les régresseurs sont endogènes, la construction du modèle logit permet de trouver la fonction linéaire des variables explicatives qui séparent le mieux les classes de réponse (voir Afsa Essafi (2003)) ; à ce titre, il s'agit d'une approche descriptive donnant les poids relatifs des variables intervenant dans les décisions du Conseil. Cette méthodologie n'a cependant qu'un faible pouvoir prédictif, dans la mesure où la valeur des variables est corrélée avec la décision et ne peut donc pas être déterminée parfaitement sur un nouveau cas.

L'interprétation des coefficients $\beta$ dans un modèle logit ordonné polytomique n'est pas évidente, en particulier parce que l'effet d'une variable est non linéaire et qu'il affecte de manière différente les probabilités des trois résultats possibles. Pour donner cependant une idée de l'importance d'une variable, il est possible de regarder son effet sur la probabilité d'autorisation ou d'interdiction pour le cas moyen. La probabilité observée d'autorisation est d'environ $60 \%$ alors que la probabilité d'interdiction est de $15 \%$. Supposons que pour un cas la probabilité d'autorisation soit de $60 \%$. Si une variable fait diminuer l'indice latent d'une unité, la probabilité d'autorisation passe alors à $80 \%$. Au contraire une variable qui augmente l'indice latent d'une unité fait passer la probabilité d'interdiction de $15 \%$ à $32 \%$. 


\subsection{Description des avis : poids des différentes variables}

Dans un premier temps, nous avons cherché à décrire le poids des différentes variables utilisées par le Conseil pour justifier ses avis au moyen d'un indice linéaire.

\subsubsection{La variable puissance d'achat}

La variable puissance d'achat est parfaitement corrélée avec l'avis du Conseil, puisque dans les 19 cas où elle est mentionnée, le Conseil a proposé une autorisation sans conditions (voir les statistiques descriptives dans l'annexe A.3). Il semble que la présence de clients disposant d'un fort pouvoir de négociation soit une raison suffisante pour entraîner l'absence de problème de concurrence lors d'une concentration. Le raisonnement du Conseil est que le secteur aval dispose de suffisamment de pouvoir pour maintenir les prix au niveau concurrentiel.

Le « poids » de cette variable est infini dans un modèle logistique. Pour analyser le poids des autres variables, il faut se restreindre aux cas où la puissance d'achat n'est pas mentionnée.

\subsubsection{Les variables barrières à l'entrée et monopole}

Nous avons appliqué la procédure logistique présentée précédemment aux 47 cas restants, qui comportent 9 interdictions, 17 autorisations sous conditions et 21 autorisations.

Les deux variables barrières à l'entrée et monopole sont significatives. La mention de barrières à l'entrée et la création d'un monopole ou quasi-monopole augmentent significativement la probabilité d'une interdiction ${ }^{19}$ (voir la régression dans l'annexe B.1). Ces deux variables sont celles qui ont le plus fort pouvoir descriptif des avis du Conseil, après conditionnement selon la puissance d'achat. Il s'agit des seules variables significatives au seuil de $10 \%$.

\subsection{Explication des avis du Conseil}

La modélisation précédente montre le pouvoir descriptif des variables de puissance d'achat et de présence de barrières à l'entrée. Cependant, ces deux phénomènes ne font pas l'objet d'une mesure objective dans les avis du Conseil : il s'agit de variables qualitatives sur lesquelles le Conseil dispose d'une certaine latitude. Il est plausible que la mention de la présence de barrières à l'entrée ne corresponde pas toujours à l'énoncé d'un fait mais corresponde plutôt à un choix du Conseil pour justifier sa décision. Dans ce cas, la variable barrières à l'entrée est endogène car corrélée avec la variable d'erreur du modèle.

Pour expliquer les avis du Conseil, nous avons choisi de nous restreindre à des variables dont nous pensons qu'elles sont exogènes. Ces choix sont discutables, comme nous l'avons développé précédemment à propos des parts de marché.

\subsubsection{Les parts de marché}

Les variables permettant d'expliquer l'avis du Conseil sont les variables de parts de marché acquéreur et cible avec un coefficient significativement positif

\footnotetext{
19 Si la probabilité d'interdiction est de $15 \%$ sans barrières à l'entrée ou monopole, elle passe à environ $60 \%$ en leur présence.
} 
(au seuil de $10 \%$ ). Une forte part de marché augmente bien la probabilité d'interdiction, dans les mêmes proportions pour la part de l'acquéreur ou de la cible $^{20}$.

\subsubsection{Les variables sectorielles}

Des indicatrices sectorielles ont également un impact significatif sur l'avis du Conseil : le secteur des infrastructures a une probabilité plus forte d'interdiction, alors que le secteur industrie a une probabilité plus forte d'autorisation (significatif au seuil de $5 \%{ }^{21}$.

Ceci peut tenir au fait que ces secteurs sont réputés pour leurs pratiques anticoncurrentielles, comme le Conseil le rappelle lui-même lors de l'acquisition du Groupe GTM par la société Vinci ${ }^{22}$ « [le] Conseil ne peut, à cet égard, que constater qu'il est saisi, de manière récurrente de pratiques d'ententes entre les principaux opérateurs de ce secteur ». Ces facteurs peuvent expliquer en partie les réticences du Conseil.

En sens inverse, ce dernier tolère peut-être une plus grande concentration dans les secteurs industriels, où les parts de marché sont souvent mesurées au niveau national mais où la possibilité d'entrée d'autres acteurs européens ou mondiaux est envisagée. Cet aspect est souvent mentionné par les barrières à l'entrée, présentes dans $51 \%$ des cas hors industrie et dans seulement $22 \%$ des cas dans l'industrie.

\subsubsection{Les variables de personnalité}

Nous avons envisagé des modélisations dans lesquelles ont été introduites des indicatrices portant sur l'identité du président de la séance ou sur celle du ministre de l'Économie à l'époque de l'opération (pour les ministres ou présidents concernés par plusieurs avis).

Les coefficients des variables relatives au président de séance ou à l'identité du ministre de l'Économie ne sont significatifs dans aucune des modélisations testées. Autrement dit, ni le président ni le ministre n'exercent d'influence significative sur l'avis rendu, ou du moins l'influence que l'un ou l'autre peut exercer est inférieure aux effets économiques que nous avons introduits. De même, la variable droite n'est pas significative.

Bergman, Jakobsson, et Razo (2005) et Lauk (2003) n'ont pas mis en évidence d'effet significatif des variables «politiques ». Nos résultats vont également dans ce sens.

\subsubsection{Explication des décisions du ministre}

Il est possible d'utiliser les mêmes variables pour expliquer les décisions du ministre de l'Économie ${ }^{23}$. Comme il a été indiqué dans le tableau 1, il existe

\footnotetext{
20 Si la probabilité d'interdiction est initialement de $15 \%$, elle passe à environ $22 \%$ pour une augmentation de part de marché de $20 \%$.

21 Si la probabilité d'interdiction est initialement de $15 \%$, elle passe à $50 \%$ si le secteur des infrastructures est concerné. Si la probabilité d'autorisation est initialement de $60 \%$, elle passe à $90 \%$ si le secteur de l'industrie est concerné.

22 Avis $\mathrm{n}^{\circ}$ 01-A-08.

23 Les autorisations tacites ont été regroupées avec les autorisations.
} 
peu de différences entre les décisions et les avis, aussi est-il normal de retrouver les mêmes variables significatives avec des coefficients sensiblement équivalents (voir les résultats en annexe dans la table 8). On peut cependant noter que les coefficients des parts de marché sont plus forts et que la régression est plus significative (en utilisant par exemple le pseudo- $R^{2}$ ) que pour la régression équivalente sur les avis. Le ministre est plus sensible aux parts de marché. Par exemple, si on reprend le cas de divergence entre le Conseil et le ministre Sara Lee-Benckiser, on constate que le Conseil a suggéré une autorisation sans condition malgré une part de marché cumulée de $85 \%$, alors que le ministre a interdit l'opération.

\subsection{Robustesse des estimations par les méthodes du jackk- nife et du bootstrap}

L'estimation du modèle par la méthode du maximum de vraisemblance permet d'obtenir des écarts-types pour les différents paramètres, ainsi que des tests de significativité des paramètres de type Wald ou $\chi^{2}$. Cependant, ces procédures de test s'appuient sur des intervalles de confiance asymptotiques, alors que nous sommes très loin de ce régime.

Aussi avons-nous mis en œuvre des méthodes de simulation adaptées à la taille de notre échantillon : les méthodes du jackknife et du bootstrap.

Nous avons appliqué celles-ci à l'estimation de notre modèle de référence, utilisant comme variables explicatives les parts de marché de la cible et de l'acquéreur ainsi que les indicatrices sectorielles infrastructures et industrie.

Après avoir eu recours à la procédure du jackknife dont les résultats sont présentés dans l'annexe B.2.3, nous avons procédé à 10000 réplications bootstrap de notre base pour estimer la fonction de répartition des différents paramètres. Les résultats sur la distribution des paramètres sont présentés dans le tableau 2 .

\begin{tabular}{lrrr}
\hline \hline & \multicolumn{3}{c}{ Intervalle de confiance 90 \% } \\
& Valeur Logit & $-4,81$ & $-2,03$ \\
Seuil $s_{1}$ & $-3,05$ & $-2,53$ & $-0,36$ \\
Seuil $s_{2}$ & $-1,32$ & 0,35 & 5,38 \\
Acquéreur & 2,48 & $-0,20$ & 5,01 \\
Cible & 2,23 & 0,94 & 2,80 \\
Infrastructure & 1,71 & $-3,47$ & $-0,46$ \\
Industrie & $-1,59$ & & \\
\hline
\end{tabular}

TAB. 2 - Estimation par bootstrap

Les intervalles de confiance montrent la faible précision des résultats mais ils fondent une certaine robustesse du modèle proposé.

\section{$5 \quad$ Analyse boursière : quelques exemples}

Nous présentons quelques exemples de réaction des marchés boursiers à l'annonce d'opérations de concentration, autre approche possible de l'évaluation des 
décisions des autorités de concurrence. Au préalable, nous rappelons le principe des études d'événement, leurs fondements théoriques ainsi que leurs limites.

\subsection{Les études d'événement et l'efficacité des marchés fi- nanciers}

Ce type d'étude repose sur le principe suivant : si une concentration est pro-compétitive, autrement dit si elle aboutit in fine à une baisse du prix, elle diminue les profits anticipés des entreprises concurrentes et donc leur cours de bourse. Au contraire, une opération anti-concurrentielle va se traduire par une hausse des prix et donc des profits des entreprises concurrentes, d'où une hausse du cours de bourse, sous l'hypothèse que le cours de bourse d'une entreprise correspond à la valeur actualisée de ses profits futurs ${ }^{24}$. L'espoir est qu'en analysant la réaction des marchés financiers à l'annonce d'une opération de concentration, il soit possible d'en déduire son effet concurrentiel anticipé ${ }^{25}$.

Afin de tester cette intuition, on estime la relation existant entre le cours de bourse et un indice de référence en période «normale », c'est-à-dire avant l'annonce de rapprochement. Puis on cherche à voir si cette relation est ou non modifiée aux alentours de la date de l'opération. On nomme mouvement « anormal » toute modification susceptible de survenir lors de cette période. Ce type d'approche est connu sous le nom d'étude d'événement.

MacKinlay (1985) rappelle que la majorité des études d'événement portant sur les acquisitions établissent que les rendements anormaux pour la cible sont élevés et positifs, tandis qu'ils sont proches de zéro pour l'acquéreur. Ce fait stylisé - une prime accordée à la cible alors que l'acquéreur est souvent pénalisé - est l'un des traits marquants des fusions et acquisitions. Les acquéreurs surestiment-ils en moyenne la valeur de leur cible? Le débat n'est toujours pas tranché ${ }^{26}$.

\subsubsection{Les limites matérielles}

Cette méthode suppose tout d'abord que les entreprises soient cotées de façon liquide sur un marché. Au cas d'espèce, il s'agit d'une limitation forte car le nombre de cas traités par le Conseil de la concurrence pour lesquels les parties et tout ou partie de leurs concurrents remplissent cette condition est restreint.

De plus, il doit exister une date identifiable à laquelle l'opération a été rendue publique, ce qui n'est pas toujours le cas.

Enfin, lorsque l'opération concerne une filiale appartenant à un grand groupe et que seul ce dernier est coté, l'analyse de son cours de bourse peut ne pas être satisfaisante. D'une part ce dernier dépend d'informations n'ayant pas de rapport avec l'opération étudiée. D'autre part, le chiffre d'affaires concerné par

\footnotetext{
24 Fama (1970) définit l'efficience semi-forte pour un marché par l'intégration de toute information publique dans les prix. La notion plus forte d'efficacité forte suppose l'intégration de toute information privée. Sa conclusion est que l'efficience semi-forte est généralement validée par les données empiriques, alors que l'efficience forte, quant à elle, est plutôt vue comme «a benchmark against which deviations from market efficiency (interpreted in its strictest sense) can be judged».

25 Il existe une large littérature concernant les études d'événement et leur utilisation pour évaluer la politique de la concurrence (voir par exemple Eckbo (1985)).

26 Voir Andrade, Mitchell, et Stafford (2001) pour une analyse récente de cette question.
} 
l'opération par rapport au chiffre d'affaires global du groupe peut être très faible, ce qui fait que l'opération n'a qu'un impact mineur sur le cours de bourse.

\subsubsection{Les problèmes des anticipations}

Une difficulté de cette méthodologie est qu'elle suppose une annonce d'opération n'ayant été précédée d'aucune rumeur, «fuite» ou anticipation. Malatesta et Thompson (1985) proposent un modèle de réaction des marchés financiers à des événements partiellement anticipés qu'ils utilisent pour estimer la valeur d'achat lors de fusions et acquisitions. Si l'événement est partiellement anticipé, l'impact économique réel est plus élevé que l'effet d'annonce, comme le vérifie l'étude économétrique des auteurs.

De manière plus générale, une concentration n'est pas un phénomène totalement exogène, elle a lieu dans un secteur dans lequel peuvent exister des anticipations de concentration selon différentes configurations. Fridolfsson et Stennek (2004) proposent une analyse de l'effet out-of-play dans lequel l'annonce d'une opération de concentration particulière exclut d'autres opérations concurrentes préalablement anticipées par les marchés financiers. Dans ce cas, les mouvements observés peuvent correspondre à une actualisation des anticipations indépendante des effets anti-concurrentiels. Le cours d'un concurrent peut baisser non parce que l'opération est pro-concurrentielle mais parce que les marchés financiers anticipaient une autre configuration plus avantageuse pour lui.

Lorsqu'une entreprise cotée est susceptible de faire l'objet d'une offre publique d'achat (OPA), son cours de bourse peut inclure une prime qui correspond à la surcote attendue lors de ce type d'opération. Une première opération de concentration peut agir comme un signal que d'autres opérations vont suivre, ce qui tend à augmenter la prime des concurrents; il est au contraire possible que la consolidation du secteur soit terminée (par exemple parce que toute fusion ultérieure serait bloquée par les autorités de concurrence), ce qui tend à diminuer les primes des concurrents.

Il faut noter que les marchés financiers peuvent également anticiper la réaction des autorités de concurrence. Si deux entreprises proposent une fusion entraînant des problèmes évidents de concurrence et donc un risque d'interdiction, cette éventualité est intégrée dans les prix.

Par contre, une fois l'annonce rendue publique, le traitement par les autorités de concurrence d'un cas de concentration est processus long, comportant de nombreuses étapes intermédiaires et de nombreux échanges entre les entreprises et l'autorité de concurrence. En conséquence, la décision d'une autorité de concurrence est, sauf exception, intégrée par les marchés financiers sur une longue période sans l'effet de surprise permettant de l'identifier.

\subsection{Réaction des marchés boursiers : étude de quelques cas}

Sur les 66 cas de concentration traités par le Conseil, nous n'avons été en mesure d'obtenir des données boursières pertinentes que sur 12 cas, ce qui interdit toute étude statistique. Aussi présentons-nous seulement quelques cas pour illustrer des faits stylisés. 


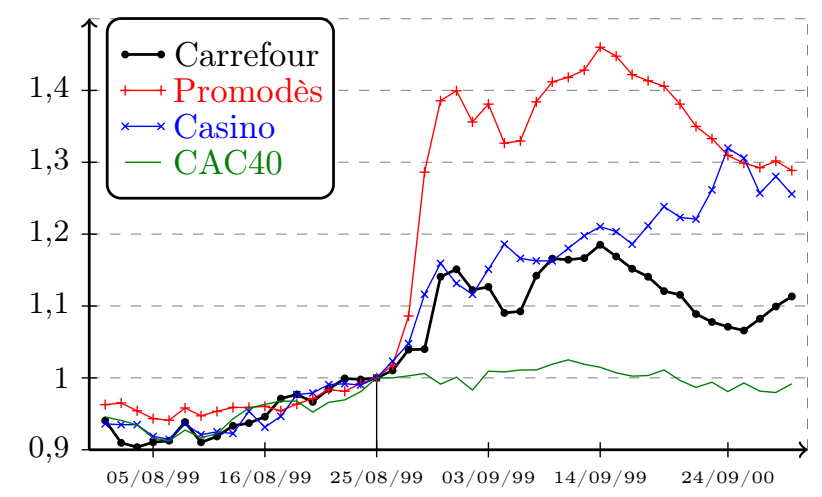

FIG. 2 - Acquisition du groupe Promodès par le groupe Carrefour (1999).

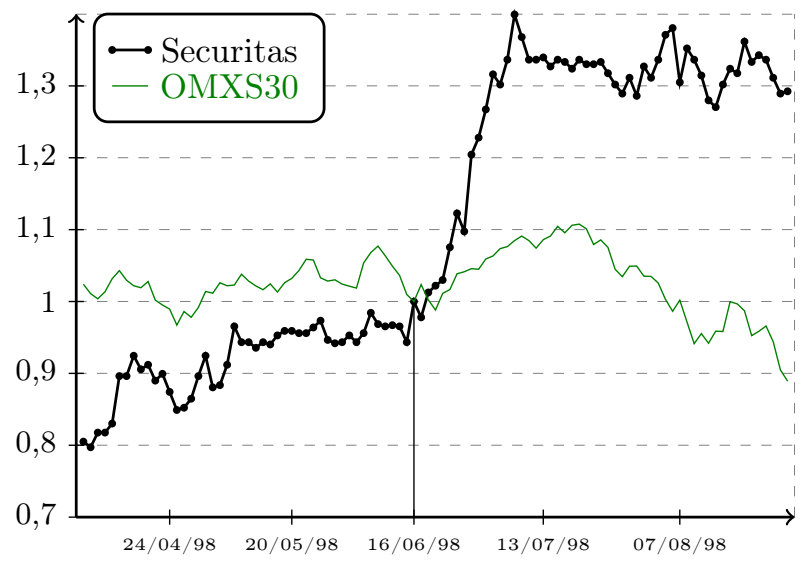

FIg. 3 - Acquisition de la société Proteg par la société Securitas AB (1998).

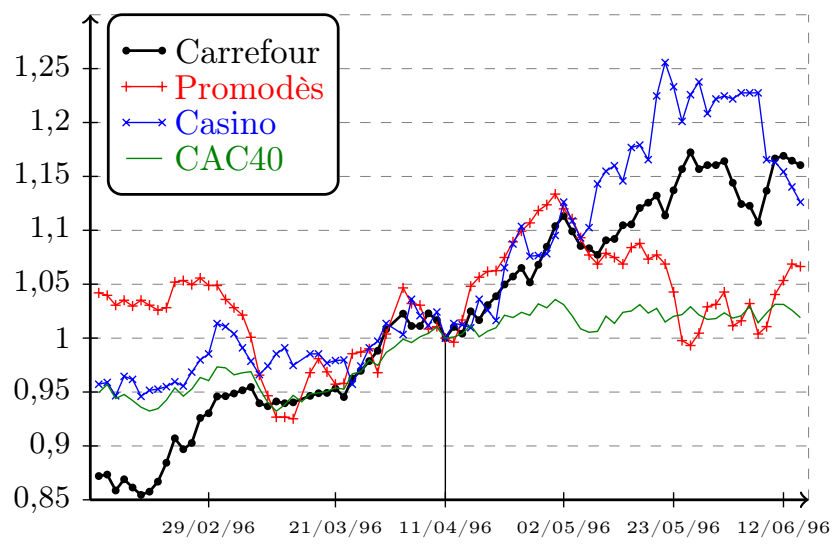

FIG. 4 - Acquisition du groupe Docks de France par le groupe Auchan (1996). 


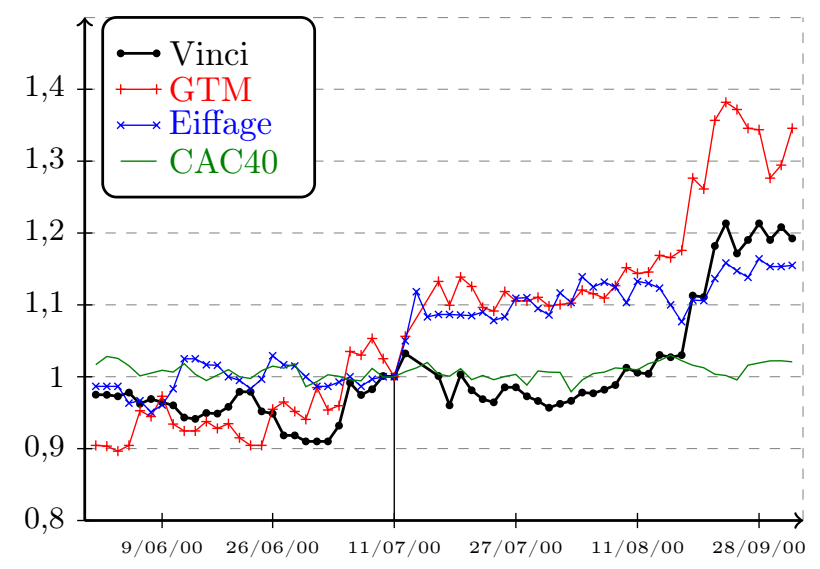

FIG. 5 - Acquisition du groupe GTM par le groupe Vinci (2000).

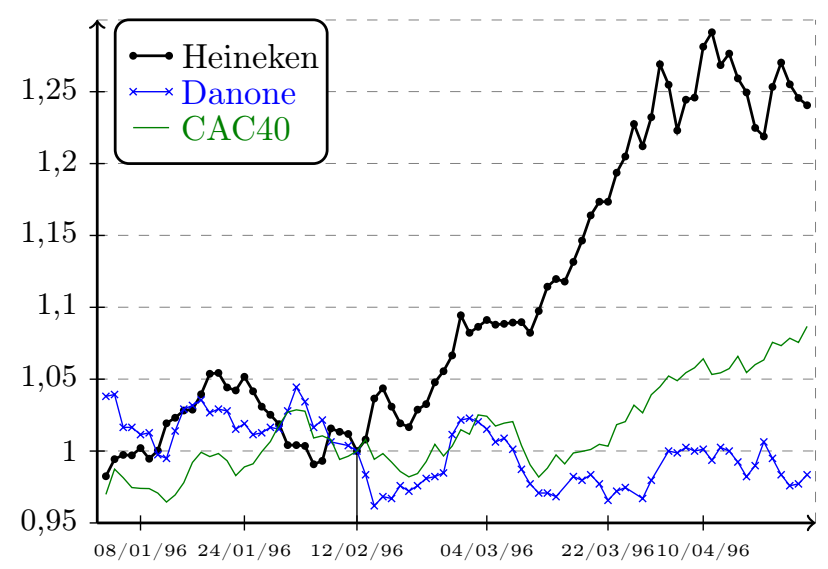

FIG. 6 - Acquisition de la société Fischer par la société Sogebra (1996). 
Concernant la représentation graphique, nous avons choisi de normaliser les cours de bourse à un le jour de l'annonce de l'opération, repérée par un trait vertical sur les figures. Un mouvement anormal est repéré par l'écart à l'indice de référence.

Nous présentons trois exemples de configuration possible, ainsi éventuellement qu'une interprétation concurrentielle. Cette interprétation est à prendre avec prudence, du fait des nombreuses critiques que nous avons mentionnées.

\subsubsection{Hausse du cours de la cible}

Le cours de l'entreprise-cible Promodès augmente fortement lors de l'annonce de son rachat par Carrefour (figure 2), ce qui illustre l'un des faits stylisés les plus robustes de l'étude des fusions et acquisitions.

\subsubsection{Hausse du cours de l'acquéreur}

Le cours de la société Securitas monte à l'annonce du rachat de Proteg (figure 3). Nous avons pu identifier plusieurs autres acquisitions mineures réalisées en Europe par la société Sécuritas en Europe (Kessler en France, Segurcat en Espagne, Deutsche Sicherheit en Allemagne) au premier semestre de 1998, ainsi que le rachat de Raab Karcher Sicherheit $(R K S)$, numéro un du marché allemand de la sécurité, annoncé en juin 1998. Il y a donc un mouvement de concentration du secteur de la surveillance au niveau européen dont profite $S e$ curitas. L'effet du seul rachat de Proteg ne peut être séparé des autres opérations concomitantes.

\subsubsection{Mouvement du cours d'un concurrent}

Dans toutes les opérations dans le secteur de la grande distribution, il existe toujours au moins un concurrent dont le cours augmente lors de l'annonce. Dans les deux cas présentés ici, le cours de Casino augmente alors que l'entreprise ne participe pas à l'opération (figures 2 et 4 ). Ceci peut signifier que les marchés anticipent des profits plus élevés dans un secteur plus concentré, ce qui traduit une opération anti-concurrentielle. Mais il peut également s'agir d'anticipation d'autres opérations à venir, dans lesquelles Casino serait une cible et disposerait de la prime associée à une entreprise OPAble.

Lors de l'annonce de l'acquisition du groupe GTM par le groupe Vinci (figure 5), le cours de leur concurrent Eiffage a augmenté et s'est maintenu par la suite à des niveaux plus élevés que le CAC 40.

Nous pouvons supposer que les marchés anticipent ici une opération anticoncurrentielle, ou du moins que le groupe Eiffage tire également profit de l'opération. Si la hausse du cours d'Eiffage provient du renforcement du risque de collusion suite à l'opération, il y a une divergence avec l'avis du Conseil qui a autorisé l'opération.

Pour finir cette section, il nous semble intéressant de remarquer que le cours de Danone, concurrent direct d'Heineken sur le marché de la bière avec sa filiale Kanterbrau, diminue lors de l'annonce du rachat de Fischer par Sogebra, filiale d'Heineken (figure 6). Précisons que la vente de cette boisson constitue au moment de l'opération environ $10 \%$ du chiffre d'affaires de Danone, ce qui peut justifier un impact de cette activité sur le cours de bourse. 
Si l'on accepte l'interprétation concurrentielle du choc, cela signifie que les marchés financiers anticipent que l'opération se déroule aux dépens de Danone; au contraire, dans son avis ${ }^{27}$, le Conseil craint que la nouvelle configuration ne donne lieu à des comportements collusifs sur le marché de la bière. Nous sommes donc en présence de deux analyses potentiellement contradictoires de la situation.

\section{Conclusion}

L'objet de notre travail a consisté à faire le point sur la pratique française en matière de contrôle des concentrations en améliorant la lisibilité des avis du Conseil de la concurrence. L'analyse économétrique, nécessairement limitée par la faible taille de notre échantillon, permet de mettre en lumière un certain nombre de résultats : les variables sur la nature de la concurrence, comme les barrières à l'entrée, la puissance d'achat, la création d'un monopole, semblent les plus déterminantes dans l'appréciation du Conseil.

Ce résultat, qui s'écarte des modèles standards de concurrence oligopolistique mettant en avant l'importance des parts de marché, est cependant cohérent avec les prédictions de modèles plus évolués, qui montrent que l'impact «social» d'une concentration ne dépend pas uniquement de facteurs structurels comme les parts de marché, mais résulte des interactions stratégiques entre les différents acteurs, les concurrents, les consommateurs, le marché en amont et en aval et les entrants potentiels. Ce résultat est conforme avec la présence explicite dans les textes juridiques français de la prise en compte de la contribution au progrès économique, qui ne se retrouve pas dans les textes européens plus attentifs à la protection de la concurrence.

Cependant cette approche essentiellement descriptive est limitée par le biais qui intervient dans la définition des variables.

Une régression plus robuste se limitant à des variables « objectives » permet de faire réapparaître le rôle des parts de marché ainsi que celui de certains secteurs : le secteur de l'industrie fait l'objet d'un contrôle moins strict, alors que des secteurs connus pour leurs pratiques anti-concurrentielles font l'objet d'un contrôle plus strict.

Les limites de notre travail sont nombreuses. Il ne concerne qu'un nombre restreint de cas, sur lequel l'analyse quantitative laisse une part certaine à l'arbitraire. Pour ne pas nous retrouver avec un échantillon de taille trop faible et biaisé dans le temps, nous avons eu recours à un certain nombre de proxies, en fonction des données disponibles dans les avis.

Les perspectives qui s'ouvrent maintenant sont diverses. Notre travail suppose une certaine homogénéité de la loi ; après quelques années d'application de la loi NRE en France, il sera possible d'apprécier si cette dernière a introduit des changements significatifs. Une piste intéressante peut être une comparaison plus précise des avis du Conseil de la concurrence avec l'analyse qui est faite par la DGCCRF. En France, cette structure particulière introduit des biais, puisque tous les cas importants ne sont pas nécessairement examinés par le Conseil, les services du ministre pouvant négocier directement des engagements depuis la

\footnotetext{
27 Avis no 96-A-09.
} 
loi NRE. La notification obligatoire a introduit aujourd'hui un travail supplémentaire pour la DGCCRF mais elle peut éventuellement permettre à l'avenir des études exhaustives sur les concentrations en France. 


\section{Références}

Afsa EssAfi, C. (2003): "Les modèles logit polytomiques non ordonnés : théorie et applications," Document de travail 0301, INSEE.

Allain, M.-L., ет S. Souam (2004): "Concentrations horizontale et relations verticales," Cahiers du Laboratoire d'économétrie no 2004-02, École Polytechnique.

Andrade, G., M. Mitchell, et E. Stafford (2001): "New Evidence and Perspectives on Mergers," Journal of Economic Perspectives, 15(2), 103-120.

Baumol, W. J., J. C. Panzar, et R. D. Willig (1982): Contestable Markets and the Theory of Industry Structure. Hartcourt Brace Jovanovich, San Diego.

Bergman, M. A., M. Jakobsson, et C. Razo (2005): "An Econometric Analysis of the European Commission's Merger Decisions," International Journal of Industrial Organization, 23(9-10), 717-737.

Cameron, A. C., et F. A. G. Windmeijer (1997): "An R-Squared Measure of Goodness of Fit for Some Common Nonlinear Regression Models," Journal of Econometrics, 77(2), 329-342.

CE 1310/97 (1997): "Règlement (CE) no 1310/97 du Conseil du 30 juin 1997 modifiant le règlement $(\mathrm{CEE}) \mathrm{n}^{\mathrm{o}} 4064 / 89 \mathrm{du}$ Conseil relatif au contrôle des concentrations entre entreprises," Journal officiel des communautés européennes, L 180, 1-6.

CE 1310/97 corrigendum (1998): "Corrigendum au règlement $(\mathrm{CE}) \mathrm{n}^{\mathrm{o}}$ 1310/97 du Conseil du 30 juin 1997 modifiant le règlement (CEE) n ${ }^{\circ}$ 4064/89 du Conseil relatif au contrôle des concentrations entre entreprises," Journal officiel des communautés européennes, L 40, 17.

CE 139/2004 (2004): "Règlement (CE) no 139/2004 du Conseil du 20 janvier 2004 relatif au contrôle des concentrations entre entreprises," Journal officiel des communautés européennes, L 24, 1-22.

CEE 4064/89 (1989): "Règlement (CEE) no 4064/89 du Conseil du 21 décembre 1989 relatif au contrôle des concentrations entre entreprises," Journal officiel des communautés européennes, L 395, 1-12.

Compte, O., F. Jenny, et P. Rey (2002): "Capacity Constraints, Mergers and Collusion," European Economic Review, 46(1), 1-29.

Deneckere, R., et C. Davidson (1985): "Incentives to Form Coalitions with Bertrand Competition," RAND Journal of Economics, 16(4), 473-486.

Derhy, A. (1995): "Les fusions et acquisitions en France de 1959 à 1992 : évolution et caractéristiques," Revue d'Économie Industrielle, 73, 19-44.

Eскво, B. E. (1985): "Mergers and the Market Concentration Doctrine: Evidence from the Capital Market," Journal of Business, 58(3), 325-49.

Estrella, A. (1998): "A New Measure of Fit for Equations with Dichotomous Dependent Variables," Journal of Business and Economic Statistics, 16(2), 198-205. 
FAmA, E. F. (1970): "Efficient Capital Markets: A Review of Theory and Empirical Work," The Journal of Finance, 25, 383-417.

Farrell, J., et C. Shapiro (1990): "Horizontal Mergers: An Equilibrium Analysis," American Economic Review, 80(1), 107-26.

Fridolfsson, S.-O., et J. StenneK (2004): "Industry Concentration and Welfare - On the Use of Stock Market Evidence from Horizontal Merger," Working paper, Research Institute of Industrial Economics, Stockholm.

FudenberG, D., et J. Tirole (1984): "The Fat-Cat Effect, The Puppy-Dog Ploy, and the Lean and Hungry Look," American Economic Review, 74(2), $361-366$.

JENNy, F. (1994): "Competition and Efficiency," in Antitrust in a Global Economy, édité par F. U. L. School. Corporate Law Institute, Transnational Juris Kluwer.

Khemani, R. S., et D. M. Shapiro (1993): "An Empirical Analysis of Canadian Merger Policy," Journal of Industrial Economics, 41(2), 161-77.

Lauk, M. (2003): "Econometric Analysis of the Decisions of the German Cartel Office," Jahrbücher für National ökonomie und Statistik, 223(6), 680-711.

MacKinlay, C. A. (1985): "Event Studies in Economics and Finance," Journal of Economic Literature, 35(1), 13-39.

Malatesta, P. H., et R. Thompson (1985): "Partially Anticipated Events: A model of Stock Price Reactions with an Application to Corporate Acquisitions," Journal of Financial Economics, 14, 237-250.

MC FAdden, D. L. (1974): "Conditional Logit Analysis of Qualitative Choice Behavior," in Frontiers in Econometrics, édité par P. Zarembka, pp. 105-142. Academic Press, New York.

Mc Fetridge, D. (1996): "The Efficiencies Defense in Merger Cases," in The Economics of the Antitrust Process, édité par M. Coate, and A. K. eds., pp. 89-115. Kluwer Academic Publishers, Boston.

Motta, M. (2004): Competition Policy. Cambridge University Press, Cambridge.

Rey, P., et J. Tirole (2005): "A Primer on Foreclosure," in Handbook of Industrial Organization, édité par M. Armstrong, and R. Porter, vol. 3. NorthHolland, Amsterdam.

Williamson, O. (1968): "Economies as an Antitrust Defense: The Welfare Trade-Offs," American Economic Review, 58, 18-36. 


\section{A Les variables explicatives}

Les variables utilisées dans les régressions sont détaillées dans cette section.

\section{A.1 Les variables exogènes}

Chiffre d'affaires : variable indicatrice valant 1 si le seuil absolu de chiffre d'affaires est dépassé pour l'opération.

Défaillante : variable indicatrice valant 1 si l'une des entreprises parties à la concentration est proche de la faillite.

Marchés locaux : variable indicatrice valant 1 si le Conseil a retenu une définition locale des marchés.

Acquéreur : la part de marché de l'entreprise acheteuse sur le marché pertinent retenu.

Cible : la part de marché de l'entreprise acquise sur le marché pertinent retenu.

Cumulée : la part de marché cumulée des parties à l'opération sur le marché pertinent retenu.

Monopole : variable indicatrice valant 1 si la part de marché cumulée des entreprises parties à l'opération est supérieure à $90 \%$.

Indicatrices sectorielles : variables indicatrices du secteur d'activité concerné. Les secteurs sont précisés dans l'annexe A.4.

Droite : variable indicatrice valant 1 si le ministre de l'Économie est de droite au moment où le Conseil rend son avis, 0 sinon.

Grande distribution : variable indicatrice valant 1 si un produit concerné par la concentration est vendu principalement par l'intermédiaire de la grande distribution.

Concurrent équivalent : variable indicatrice valant 1 s'il existe sur le marché pertinent une entreprise ayant une structure comparable à l'entité issue de la fusion.

En ce qui concerne les parts de marché, si l'avis fait part d'une présence marginale sur le marché, nous avons estimé que cela signifiait que la part de marché était comprise entre 0 et $5 \%$. En outre, si l'avis n'indique qu'une plage de valeur (en raison notamment du secret des affaires, ou d'une définition plus ou moins large du marché), nous avons pris le parti de retenir le centre de l'intervalle. Enfin, il existe une relation linéaire entre ces trois variables :

$$
\text { Cumulée }=\text { Acquéreur }+ \text { Cible. }
$$

\section{A.2 Les variables endogènes}

Barrières : indicatrice prenant la valeur 1 si l'existence de barrières à l'entrée sur le marché considéré est mentionnée dans l'avis.

Puissance d'achat : variable indicatrice prenant la valeur 1 si l'avis fait état de l'existence d'entreprises en amont ou en aval disposant d'un pouvoir de négociation important, capables de contraindre la politique de prix sur le marché pertinent.

Collusion : indicatrice prenant la valeur 1 si le Conseil mentionne la présence ou le risque de collusion. 


\section{A.3 Statistiques descriptives simples}

Pour les variables de part de marché, exprimées entre 0 et 100, voir table 3. Entre parenthèses figure l'écart-type.

\begin{tabular}{lcccc}
\hline \hline & & \multicolumn{3}{c}{ Par catégorie } \\
\cline { 3 - 5 } & Moyenne & Autorisation & Sous conditions & Interdiction \\
\hline Cumulée & 50 & 51 & 42 & 66 \\
Acquéreur & $(26)$ & $(24)$ & $(29)$ & $(21)$ \\
& 26 & 25 & 25 & 34 \\
Cible & $(21)$ & $(20)$ & $(20)$ & $(26)$ \\
& 24 & 25 & 17 & 33 \\
& $(23)$ & $(24)$ & $(24)$ & $(22)$ \\
\hline
\end{tabular}

TAB. 3 - Variables de part de marché

Pour les variables dichotomiques, voir table 4. La fréquence est précisée endessous.

\begin{tabular}{|c|c|c|c|c|c|}
\hline & \multirow{2}{*}{$\begin{array}{l}\text { Nombre d'observations } \\
\text { prenant la modalité } 1\end{array}$} & \multicolumn{4}{|c|}{ Par catégorie } \\
\hline & & Autorisation & Sous & conditions & Interdiction \\
\hline \multirow[t]{2}{*}{ Puissance d'achat } & 19 & 19 & & 0 & 0 \\
\hline & $29 \%$ & $48 \%$ & & $0 \%$ & $0 \%$ \\
\hline \multirow[t]{2}{*}{ Barrières } & 26 & 10 & & 8 & 8 \\
\hline & $39 \%$ & $25 \%$ & & $47 \%$ & $89 \%$ \\
\hline \multirow[t]{2}{*}{ Monopole } & 6 & 2 & & 2 & 2 \\
\hline & $9 \%$ & $5 \%$ & & $12 \%$ & $22 \%$ \\
\hline \multirow[t]{2}{*}{ Droite } & 31 & 19 & & 7 & 5 \\
\hline & $47 \%$ & $48 \%$ & & $41 \%$ & $56 \%$ \\
\hline \multirow[t]{2}{*}{ Collusion } & 6 & 3 & & 2 & 1 \\
\hline & $9 \%$ & $8 \%$ & & $11 \%$ & $11 \%$ \\
\hline \multirow[t]{2}{*}{ Marchés locaux } & 11 & 3 & & 8 & 0 \\
\hline & $17 \%$ & $8 \%$ & & $47 \%$ & $0 \%$ \\
\hline \multirow[t]{2}{*}{ Chiffre d'affaires } & 15 & 7 & & 8 & 0 \\
\hline & $23 \%$ & $18 \%$ & & $47 \%$ & $0 \%$ \\
\hline \multirow[t]{2}{*}{ Défaillante } & 5 & 4 & & 1 & 0 \\
\hline & $8 \%$ & $10 \%$ & & $6 \%$ & $0 \%$ \\
\hline \multirow[t]{2}{*}{ Concurrent équivalent } & 20 & 14 & & 5 & 1 \\
\hline & $30 \%$ & $35 \%$ & & $29 \%$ & $11 \%$ \\
\hline
\end{tabular}

TAB. 4 - Statistiques simples des variables qualitatives 


\section{A.4 Les différents secteurs}

Les secteurs sont construits pour partie par regroupement d'activités de la nomenclature d'activités française (NAF 17) :

A. Agriculture, chasse, sylviculture

B. Pêche, aquaculture, services annexes $\}$ agro-alimentaire

C. Industries extractives

D. Industrie manufacturière $\}$ industrie

E. Production et distribution d'électricité, de gaz et d'eau

F. Construction infrastructures

- J. Activités financières : n'a donné lieu à aucune opération de concentration contrôlée sur la période considérée.

- Toutes les autres activités marchandes ont été regroupées dans les services

G. Commerce; réparations automobile et d'articles domestiques

H. Hôtels et restaurants

I. Transports et communications

K. Immobilier, location et services aux entreprises

desquels nous avons extraits deux sous-catégories qui nous semblent pertinentes pour les problèmes de concurrence spécifiques qu'elles posent :

- les médias, en raison de spécificités culturelles et politiques,

- la distribution, en raison des effets concurrentiels qui lui sont prêtés.

\begin{tabular}{|c|c|c|c|c|}
\hline & Nombre de cas & Autorisation & Sous conditions & Interdiction \\
\hline Agro-alimentaire & 12 & 7 & 1 & 4 \\
\hline Industrie & 27 & 22 & 3 & 2 \\
\hline Infrastructures & 8 & 0 & 7 & 1 \\
\hline Services & 7 & 5 & 0 & 2 \\
\hline Médias & 8 & 4 & 4 & 0 \\
\hline Distribution & 4 & 2 & 2 & 0 \\
\hline
\end{tabular}

TAB. 5 - Répartition sectorielle des opérations 


\section{B Résultats des régressions}

Dans les modélisations suivantes, nous cherchons à modéliser la probabilité d'une autorisation sans condition ni engagement. Ainsi, un coefficient positif devant une variable indique que le fait de posséder la caractéristique associée à la variable augmente la probabilité d'un avis positif.

L'estimation d'un coefficient est suivie d'une étoile $\left(^{*}\right)$ si elle est significative à $10 \%$, de deux étoiles $\left({ }^{* *}\right)$ si elle est significative à $5 \%$ et de trois étoiles $\left({ }^{* *}\right)$ si elle est significative à $1 \%$.

La qualité de l'ajustement du modèle aux données peut être mesurée par le pseudo- $R^{2}=1-\log L / \log L_{0}$, où $L_{0}$ désigne la vraisemblance du modèle sans variable explicative. Cet indicateur, proposé initialement par Mc Fadden (1974) et repris par exemple par Cameron et Windmeijer (1997) comme équivalent du coefficient de détermination empirique $R^{2}$ dans le modèle linéaire, possède cependant l'inconvénient de rester relativement faible. D'autres indicateurs ont été proposés pour pallier ce défaut, par exemple par Estrella (1998)

$$
\phi_{0}=1-\left(\frac{\log L}{\log L_{0}}\right)^{-\frac{2}{n} \log L_{0}}
$$

qui fait intervenir le nombre d'observations $n$.

\section{B.1 Modélisation des avis}

Analyse des 47 cas où la variable puissance d'achat est nulle. Variables explicatives : barrières et monopole.

\begin{tabular}{lcc}
\hline \hline & Estimation & Écart-type \\
\hline Seuil 1 & $-3,38^{* * *}$ & 0,69 \\
Seuil 2 & $-0,95^{* *}$ & 0,45 \\
Monopole & $3,10^{* *}$ & 1,20 \\
Barrières & $2,66^{* * *}$ & 0,71 \\
\hline Pseudo- $R^{2}=0,22$ & & \\
$\phi_{0}=0,40$ & &
\end{tabular}

TAB. 6 - Résultat de la régression logistique sur la sous-population des opérations pour lesquelles il n'existe pas de contre-pouvoir sur le marché pertinent 


\section{B.2 Explication des avis}

Explication des avis du Conseil à partir des variables de parts de marché et de secteur

\begin{tabular}{lcc}
\hline \hline & Estimation & Écart-type \\
\hline Seuil 1 & $-3,06^{* * *}$ & 0,77 \\
Seuil 2 & $-1,32^{*}$ & 0,66 \\
Acquéreur & $2,48^{*}$ & 1,36 \\
Cible & $2,24^{*}$ & 1,28 \\
Infrastructures & $1,71^{* *}$ & 0,81 \\
Industrie & $-1,59^{* * *}$ & 0,64 \\
\hline
\end{tabular}

Pseudo- $R^{2}=0,13$

$\phi_{0}=0,23$

TAB. 7 - Explication des avis

\section{B.2.1 Explication des décisions du ministre}

Explication des décisions du ministre à partir des variables de parts de marché et de secteur. Les estimations sont faites sur 65 observations.

\begin{tabular}{lcc}
\hline \hline & Estimation & Écart-type \\
\hline Seuil 1 & $-4,35^{* * *}$ & 0,92 \\
Seuil 2 & $-1,63^{*}$ & 0,69 \\
Acquéreur & $2,98^{* *}$ & 1,50 \\
Cible & $2,87^{* *}$ & 1,27 \\
Infrastructures & $2,41^{* *}$ & 0,83 \\
Industrie & $-1,32^{* * *}$ & 0,64 \\
\hline Pseudo- $R^{2}=0,17$ & &
\end{tabular}

Pseudo- $R^{2}=0,17$

$\phi_{0}=0,27$

TAB. 8 - Explication des décisions

\section{B.2.2 Reconstitution des avis}

Grâce aux estimations des paramètres, nous pouvons déterminer une probabilité que le Conseil rende tel ou tel avis sur chacun des cas. La définition de ces probabilités est très sensible aux seuils estimés, elles doivent donc être manipulées avec prudence.

Si l'on choisit d'estimer la valeur prédite par la valeur ayant la plus forte probabilité on obtient le tableau 9 donné à titre indicatif.

Le modèle reproduit mal les interdictions. En particulier, il existe quatre cas pour lesquelles l'avis prédit est l'opposé de l'avis rendu ${ }^{28}$. Pour ces quatre cas,

\footnotetext{
28 Il s'agit des quatre cas suivants : deux interdictions 95-A-08 CEAC-Exide, 95-A-14 Knogo-Sensormatic, et deux autorisations 00-A-04 Canal Plus-Vivendi, 93-A-04 ChicobelLeroux.
} 


\begin{tabular}{lccrr}
\hline \hline & \multicolumn{3}{c}{ Avis du Conseil } \\
\cline { 2 - 4 } Prédiction du modèle & Autorisation & $\begin{array}{c}\text { Autorisation } \\
\text { conditionnelle }\end{array}$ & Opposition & Total \\
\hline Autorisation & & 8 & 6 & 53 \\
Autorisation conditionnelle & 19 & 8 & 2 & 11 \\
Opposition & 0 & 1 & 1 & 2 \\
\hline Total & 40 & 17 & 8 \\
\hline
\end{tabular}

TAB. 9 - Reconstitution des avis

la présence de barrières à l'entrée (les deux premiers) ou leur absence (les deux derniers) est l'un des arguments du Conseil pour appuyer sa décision (conformément à notre régression descriptive de la section 4.2).

\section{B.2.3 Test de robustesse par la méthode du jackknife}

Les résultats obtenus par la méthode du jackknife sont résumés dans la table 10.

\begin{tabular}{lcccc}
\hline \hline & $\begin{array}{c}\text { Coefficient } \\
\text { Logit }\end{array}$ & $\begin{array}{c}\text { Écart-type } \\
(\mathrm{MV})\end{array}$ & $\begin{array}{c}\text { Estimateur } \\
\text { Jacknife }\end{array}$ & $\begin{array}{c}\text { Écart-type } \\
\text { Jacknife }\end{array}$ \\
\hline Seuil $s_{1}$ & $-3,05$ & 0,78 & $-2,84$ & 0,81 \\
Seuil $s_{2}$ & $-1,32$ & 0,67 & $-1,27$ & 0,64 \\
Acquéreur & 2,48 & 1,36 & 2,24 & 1,55 \\
Cible & 2,23 & 1,28 & 2,17 & 1,51 \\
Infrastructure & 1,71 & 0,81 & 1,61 & 0,54 \\
Industrie & $-1,59$ & 0,64 & $-1,36$ & 0,84 \\
\hline
\end{tabular}

TAB. 10 - Estimations des paramètres par la méthode du jackknife 


\section{B.3 Utilisation d'une modélisation non ordonnée}

Même si la modélisation ordonnée des avis du Conseil se justifie, elle impose des restrictions puisque qu'un coefficient unique donne le poids d'une variable pour séparer autorisation, autorisation sous conditions et interdiction. Un modèle logit non ordonné assigne des poids différents aux différentes variables explicatives pour chacune des modalités. Ce genre de modélisation possède deux inconvénients pour notre étude. D'une part, il suppose de choisir une modalité de référence - la valeur d'un paramètre se comprenant toujours par rapport à cette modalité de référence. Ensuite, il suppose d'estimer un jeu de coefficients différents pour chaque modalité (moins la modalité de référence), ce qui multiplie le nombre de paramètres à estimer alors que nous disposons de très peu d'observations.

Si on prend comme catégorie de référence autorisation, on obtient les coefficients suivants :

\begin{tabular}{llcc}
\hline \hline & Paramètre & Estimation & $\hat{E}$ cart-type \\
\hline \multirow{3}{*}{ Sous } & Constante & $-0,07$ & 0,65 \\
conditions & Acquéreur & 0,04 & 1,69 \\
& Cible & $-1,10$ & 1,64 \\
& Industrie & $-1,64^{* *}$ & 0,74 \\
\hline \multirow{3}{*}{ Interdiction } & Constante & $-2,63^{* *}$ & 1,03 \\
& Acquéreur & $3,26^{*}$ & 1,86 \\
& Cible & $3,09^{*}$ & 1,77 \\
& Industrie & $-1,84^{*}$ & 0,92 \\
\hline Pseudo- $R^{2}=0,12$ & & &
\end{tabular}

$\phi_{0}=0,21$

TAB. 11 - Estimations des coefficients par une régression logistique non ordonnée

Le secteur industrie diminue de manière significative la probabilité d'autorisation sous condition et d'interdiction, ce qui est cohérent avec le modèle ordonné.

Par contre, les variables de parts de marché ne sont significatives que pour la catégorie interdiction, pas pour autorisation sous condition. L'effet significatif des parts de marché dans le modèle ordonné provient sans doute de l'effet dans le cas des interdictions.

Une limite forte du modèle logit non ordonné dans notre cas est qu'il nécessite de la variabilité dans chacune des catégories pour permettre une estimation or la catégorie interdiction ne comporte que neuf observations. 


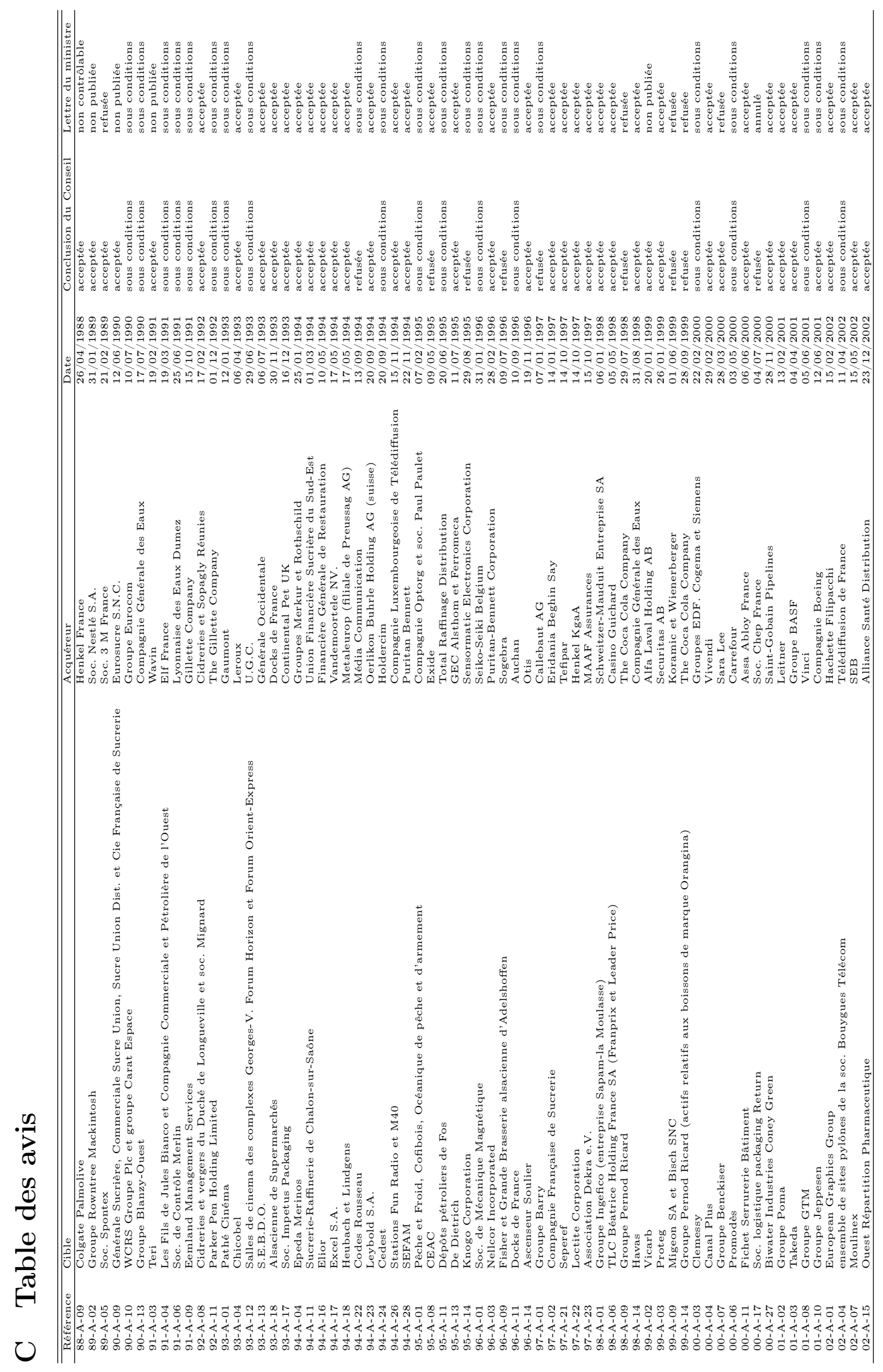

